\title{
Das Innovationsprinzip - Ein neues europäisches Rechtsprinzip?
}

\author{
Christian Calliess"
}

Inhalt
A. Einführung
126
B. Begriffliche Annäherung 128
C. Innovationssteuerung durch Recht 129
I. Einführung
II. Rechtliche Vorgaben zur Förderung von Innovationen im Unionsrecht 129
III. Regulierung von Innovationen als Auftrag an den Unionsgesetzgeber 131
IV. Zwischenergebnis 133
D. Innovationsfreiheit, Verhältnismäßigkeit und „bessere Rechtsetzung“ 134
E. Gemeinwohlverträgliches Innovationspotential durch Recht ermöglichen 136
I. Das Zusammenspiel von Genehmigung, Verhältnismäßigkeits- und
Alternativenprüfung
$\begin{array}{ll}\text { II. Die Alternativenprüfung als Instrument einer innovationsoffenen } & \\ \text { Regulierung im europäischen Umweltrecht } & 137\end{array}$
III. Weitergehende Perspektiven einer Kopplung von Innovation und
Alternativenprüfung
1. Förderung von technischer und stofflicher Innovation 140
2. Alternativenprüfung de lege ferenda 140
F. Ausblick 141

\begin{abstract}
Gerade die enormen Herausforderungen der Gegenwart - Digitalisierung und Dekarbonisierung - verlangen nach mitunter tiefgreifenden Innovationen. In rechtlicher Perspektive ist in den europäischen Grundrechten der Forschungs- und Wirtschaftsfreiheit ein Innovationsprinzip enthalten, das freilich nicht als Gegenprinzip zum Vorsorgeprinzip verstanden werden kann, sondern vielmehr mit diesem im Rahmen der Verhältnismäßigkeit in Ausgleich gebracht werden muss. Auf dieser Basis ist es

* Professor für Öffentliches Recht und Europarecht an der Freien Universität Berlin (Deutschland) und Inhaber eines Ad Personam Jean Monnet Chairs. E-Mail: europarecht@fu-berlin.de. Der Beitrag ist meinem akademischen Lehrer Prof. Dr. Dr. Georg Ress zu seinem 85. Geburtstag in herzlicher Verbundenheit gewidmet. Er basiert auf dem Vortrag, den ich im Rahmen des zu Ehren von Georg Ress veranstalteten Kolloquiums am 18. Januar 2020 am Europa-Institut der Universität des Saarlandes gehalten habe.
\end{abstract}


Aufgabe des EU-Gesetzgebers, seine Regulierung im Rahmen der europäischen Better-Regulation-Agenda innovationsoffen auszugestalten. Er hat im Lichte des Verhältnismäßigkeitsprinzips zu prüfen, welche Form der Regulierung eine innovationsoffene, aber zugleich vorsorgeorientierte Politik angemessenen verwirklicht. Durch Alternativenprüfungen und Mechanismen experimenteller Gesetzgebung können erwünschte Innovationen angereizt und gelenkt werden.

\section{The Innovation Principle - A New European Legal Principle?}

This article deals with the question if the so-called innovation principle is a new principle of European law. Enormous challenges of the present - digitalisation and decarbonisation - call for sometimes far-reaching innovations. From a legal perspective, the European fundamental rights of freedom of research as well as economic freedom comprise an innovation principle, which cannot be understood as a counter-principle to the precautionary principle, but rather must be balanced with it within the framework of proportionality. On this basis, it is the task of the EU to shape European legislation in accordance with his Better-Regulation-Agenda in a way that is open towards innovation. In the light of the principle of proportionality, the European legislator must examine which form of regulation adequately implements a policy that is open to innovation but at the same time oriented towards precaution. Enabling alternative tests as well as experimental regulation can stimulate and steer desired innovations.

Keywords: Digitalisation, Decarbonisation, Freedom of Research, Economic Freedom, Innovation Principle, Precautionary Principle, Better-Regulation-Agenda, Principle of Proportionality, Alternative Tests, Experimental Regulation

\section{A. Einführung}

Wenn die Rede von einem Innovationsprinzip ist, dann handelt es sich um einen auf europäischer Ebene kontrovers diskutierten Begriff, der von einigen Akteuren insbesondere aus der Wirtschaft als Gegengewicht zum Vorsorgeprinzip verstanden wird. ${ }^{1}$ Von dieser Seite wird befürchtet, dass das Vorsorgeprinzip die Entwicklung neuer Technologien behindern und das Innovationspotenzial der europäischen Wirtschaft beschädigen könne. Daraus erwächst die Forderung, ein Innovationsprinzip in die Verträge der Europäischen Union (EU) einzuführen. Demgegenüber hat bereits die Erwähnung des Innovationsprinzips in Texten zum neuen Forschungsprogramm der EU, „Horizon Europe“, zu Protesten von Nichtregierungsorganisationen geführt, die - ebenso wie einige Mitgliedstaaten der EU - dadurch eine Schwächung des Vor-

1 Business Europe/ European Risk Forum/ European Round Table of Industrialists, Better Framework for Innovation, Fuelling EU policies with an Innovation Principle, June 2015: "Whenever EU institutions consider policy or regulatory proposals, impact on innovation should be fully assessed and addressed." 
sorgeprinzips befürchten. Das vorgeschlagene Innovationsprinzip soll nach Auffassung seiner Befürworter dazu beitragen, die europäische Gesetzgebung innovationsfreundlicher auszugestalten. Regulierung wird aus dieser Perspektive allein als Hemmnis für Innovationen betrachtet. Eine solche Sichtweise ist allerdings verkürzend: Neue Ansätze der Transformationsforschung haben anhand von Innovationsprozessen gezeigt, dass es oftmals nicht ausreichend ist, Innovationen zu fördern, sondern dass insbesondere in der Übergangsphase der Innovation von der Nische in den breiten Markt eine flexible politisch-regulative Steuerung durch den Staat erforderlich ist, die einen stabilen Rahmen schafft, der den Unternehmen die notwendige Planungs- und Investitionssicherheit vermittelt. Dies geschieht überwiegend durch Regulierung, die daher auch ein Treiber von Innovationen sein kann. Nicht zuletzt können durch Regulierung erwünschte Innovationen angereizt und gelenkt werden, um wichtige Gemeinwohlziele wie etwa den Umweltschutz zu fördern.

Erst auf dieser Basis hat die Europäische Kommission die Bedeutung eines stärker innovationsorientierten Acquis der EU zur Unterstützung der politischen Ziele der EU erkannt und ist zur Ansicht gelangt, dass Regulierung Innovationen unterstützen kann. ${ }^{2}$ Ein Arbeitspapier der Kommissionsdienststellen aus dem Jahr 2016 mit dem Titel „Bessere Rechtsetzung für innovationsgetriebene Investitionen“ ebnete bereits mit mehreren Beispielen den Weg zu einer proaktiven Nutzung der Regulierung, um Marktversagen zu beheben und Innovationsökosysteme zu vervollständigen. Seither haben die EU-Institutionen begonnen, sich auf ein „Innovationsprinzip“ als neuen Ansatz zu beziehen, der die Berücksichtigung von Innovationen im gesamten politischen Prozess fördern würde.

Im Zuge dessen ist in der Politik das Bewusstsein für die Bedeutung einer gut durchdachten Regulierung zur Förderung von Innovationen gewachsen. Wissenschaftliche Studien haben gezeigt, dass Regulierung, wenn sie ein angemessenes Maß an Stringenz und ein geeignetes Timing aufweist, Innovationen in Richtung gesellschaftlicher Bedürfnisse lenken kann. Regulierung kann jedoch auch innovationshemmend sein, indem sie z.B. aktuelle technologische Trends nicht berücksichtigt, keine Anreize für Investitionen in Forschung und Entwicklung schafft, das Entstehen alternativer Geschäftsmodelle behindert oder übermäßige Bürokratie auferlegt, die letztlich Ressourcen von produktiveren Verwendungen ablenkt.

Ein bekanntes Beispiel ist die europaweite Regulierung von Straßenbeleuchtung, die mit ihren starren Standards über lange Zeit verhinderte, dass energieeffizientere Techniken wie LED-Lampen zum Einsatz gebracht werden können. Aber auch die Beschleunigung der digitalen Innovation stellt die hoheitliche Regulierung vor neue Herausforderungen: Ein Beispiel ist das sogenannten „Pacing-Problem“, das agilere, anpassungsfähigere Regulierungssysteme sowie flexiblere und experimentellere Regulierungsansätze erfordert, die darauf abzielen, Lösungen zu testen und den laufen-

2 Dazu der Überblick in: European Commission, Study supporting the interim evaluation of the innovation principle, Final Report, Independent Expert Report by Renda/Simonelli, Brussels 2019, S. 8 ff. (online verfügbar). 
den technologischen und organisatorischen Veränderungen in einem ethisch verantwortungsvollen Sinne Rechnung zu tragen. ${ }^{3}$

\section{B. Begriffliche Annäherung}

Eine Innovation lässt sich als Schaffung neuer oder Umwidmung vorhandener Ressourcen, die zum Fortschritt beitragen, definieren. Diese Definition besteht aus zwei Elementen: ${ }^{4}$ Das erste Element - die Schaffung neuer Ressourcen - bezieht sich im Wesentlichen auf den Aspekt der Neuartigkeit. Letztere ist in einem weiten Sinne zu verstehen und bezieht sich nicht nur auf technische oder wissenschaftliche Innovationen. Innovation kann auch im Bereich des Marketings, des Managements von Prozessen oder der Organisation von bzw. innerhalb einer Branche entstehen. Darüber hinaus ist Innovation nicht auf Produkte und Marktprozesse beschränkt, sondern kann auch außerhalb des Marktes auftreten. Der so definierte Begriff der Innovation umfasst die Erzeugung und Verbreitung neuer Produkte, die Neuerungen von Verfahren oder von Strukturen, aber auch die Beeinflussung von sozialen Verhaltensweisen.

Das zweite Element der Definition - der Beitrag zum Fortschritt - enthält ein teleologisches Kriterium: Eine technische Neuheit oder ein neuer Ansatz werden nur dann als innovativ angesehen, wenn sie einen wirtschaftlichen und gesellschaftlichen Nutzen bringen. Basierend auf dieser Definition wird „Innovation“ als ein positives Ereignis betrachtet, das zum Fortschritt beiträgt und einen gesellschaftlichen Mehrwert in sich trägt.

Es gibt verschiedene Wege, auf denen eine Innovation bewirkt werden kann. Eine „Push-Innovation“ ist eine von einem Unternehmen in die Praxis eingebrachte Idee, die dann vom Markt und damit von der Gesellschaft akzeptiert werden muss. Im Gegensatz dazu reagiert eine „Pull-Innovation“ auf eine explizite Nachfrage des Marktes oder der Gesellschaft. Da letzteres eher selten der Fall ist, wird Innovation in der Regel im Sinne einer „Push-Innovation“ verstanden. Eine Innovation muss also in die reale Welt gepusht werden. Im Hinblick auf das Etablierte erscheint ihre Neuheit als Anomalie. Deshalb muss sich jede Innovation durchsetzen, sie muss die Gesellschaft überzeugen. Vor diesem Hintergrund ist eine Innovation als Prozess zu verstehen, in dem sich die Neuheit gegen das Etablierte durchsetzen muss. Dieser Prozess ist umso härter, je disruptiver die Auswirkungen einer Innovation sein können. Dies führt zu einer weiteren wichtigen Unterscheidung, nämlich der zwischen radikaler (disruptiver) und inkrementeller Innovation. ${ }^{5}$ Demnach beinhaltet die radikale Innovation einen technologischen Wandel und kann zu großen Veränderungen in den Basistechnologien der Wirtschaft führen. Diese Art von Innovation ist oft komplex und beinhaltet nichttechnologische Veränderungen. Im Vergleich dazu zielt die inkrementelle Innovation

3 Marchant/Allenby/Herkert.

4 Granieri/Renda, S. 4.

$5 \mathrm{OECD/European} \mathrm{Commission/Nordic} \mathrm{Innovation} \mathrm{Joint} \mathrm{Workshop,} \mathrm{The} \mathrm{future} \mathrm{of} \mathrm{Eco-In-}$ novation: The Role of Business Models in Green Transformation, 2012. 
darauf ab, bestehende Technologien oder Prozesse zu modifizieren und zu verbessern, um die Effizienz der Ressourcen- und Energienutzung zu erhöhen, ohne die zugrunde liegenden Kerntechnologien grundlegend zu verändern.

\section{Innovationssteuerung durch Recht}

\section{Einführung}

Die rechtswissenschaftliche Innovationsforschung muss keinen eigenständigen Innovationsbegriff entwickeln, vielmehr reagiert sie auf Innovationen in der Gesellschaft, um sie sodann in Bezug zur Rechtsordnung zu setzen. ${ }^{6}$ Im Zuge dessen soll nach Möglichkeiten gesucht werden, die Rechtsordnung so zu konzipieren und Recht so anzuwenden, dass technologische, ökologische, soziale und kulturelle Innovationen stattfinden können, aber möglichst nur zu solchen Folgen führen, die gesellschaftsverträglich sind oder im Interesse aller Bürger liegen. ${ }^{7}$

Hintergrund ist die Erkenntnis, dass eine Gesellschaft, die Innovationen als solche unterstützt, ohne dabei aber die unterschiedlichen Folgewirkungen in Betracht zu ziehen, ein hohes Risiko eingeht: Dieses Risiko realisiert sich dann, wenn die späteren negativen Wirkungen einer Innovation deren positiven Effekte im Ergebnis wieder „auffressen“. Ein Ziel rechtswissenschaftlicher Innovationsforschung ist es daher auch, Innovationsfolgen an den normativen Orientierungen der Gesellschaft zu messen, insbesondere an verfassungsrechtlichen Vorgaben, die aus den Grundrechten und verfassungsrechtlich verankerten Staatszielen fließen. Zur damit angesprochenen Gemeinwohlorientierung des Rechts gehört es auch, dass es den bestehenden Modernisierungsbedarf ebenso wie den Bedarf zur Sicherung des Status quo aufnimmt und im Sinne praktischer Konkordanz zum Ausgleich führt. Insofern geht es darum, unterschiedliche miteinander konfligierende Interessen so aufeinander zu beziehen, dass im Rahmen des Machbaren möglichst alle Berücksichtigungschancen haben (Optimierung). ${ }^{8}$ In der Konsequenz ist der für das Recht maßgebende Innovationsbegriff daher im Ergebnis doch ein normativ eingeordneter Innovationsbegriff. ${ }^{9}$

\section{Rechtliche Vorgaben zur Förderung von Innovationen im Unionsrecht}

Sieht das Unionsrecht die Förderung von Innovationen vor? Gibt es sogar ein im Vertrag verankertes „Innovationsprinzip“? Oder ist die Schaffung eines innovationsfreundlichen Europas eher ein Ziel mit politischem Charakter? Mehrere Aspekte im Unionsrecht lassen die Annahme zu, dass sich aus den Verträgen Bausteine eines Innovationsprinzips durch systemische und teleologische Auslegung ableiten lassen.

6 Hoffmann-Riem, Innovationsoffenheit und Innovationsverantwortung, AöR 2006/2, S. 257.

7 Ibid., S. 256.

8 Ibid., S. 267.

9 Ibid. 
Art. 3 Abs. 3 EUV in Verbindung mit Art. 173 und 179 AEUV implizieren das Ziel, einen auf Innovation basierenden „Europäischen Forschungsraum“ zu schaffen.

Die grundlegende Zielbestimmung des Art. 3 Abs. 3 EUV besagt:

„Die Union errichtet einen Binnenmarkt. Sie wirkt auf die nachhaltige Entwicklung Europas auf der Grundlage eines ausgewogenen Wirtschaftswachstums und von Preisstabilität, eine in hohem Maße wettbewerbsfähige soziale Marktwirtschaft, die auf Vollbeschäftigung und sozialen Fortschritt abzielt, sowie ein hohes Maß an Umweltschutz und Verbesserung der Umweltqualität hin. Sie fördert den wissenschaftlichen und technologischen Fortschritt."

Im Zusammenhang mit der Industriepolitik der EU wird „Innovation“ explizit erwähnt. Nach Art. 173 AEUV sorgen die Union und die Mitgliedstaaten dafür, dass die notwendigen Voraussetzungen für die Wettbewerbsfähigkeit der Industrie der Union gewährleistet werden. Zu diesem Zweck zielen ihre Maßnahmen im Einklang mit einem System offener und wettbewerbsorientierter Märkte unter anderem darauf ab, eine „bessere Nutzung des industriellen Potenzials der Politik in den Bereichen Innovation, Forschung und technologische Entwicklung “ zu fördern. In dieser Hinsicht ist wiederum Art. 179 Abs. 1 AEUV von Interesse. Er beinhaltet die Aufgabe, durch Stärkung der wissenschaftlichen und technologischen Grundlagen einen europäischen Forschungsraum zu schaffen. Dies soll die Union, einschließlich ihrer Industrie, dazu bringen, wettbewerbsfähiger zu werden.

Ungeachtet dessen sind die in der EU-Grundrechtecharta verankerten Garantien von zentraler Bedeutung: Mit der Freiheit der Wissenschaften (Art. 13), der Berufsfreiheit und dem Recht auf Arbeit (Art. 15) sowie dem Recht auf Eigentum einschließlich des geistigen Eigentums (Art.17) definiert die Charta wichtige(Vor-)Bedingungen für Innovation, da Innovation weitgehend aus frei ausgeübten wirtschaftlichen und wissenschaftlichen Aktivitäten entsteht. Der freie Wettbewerb und die Koexistenz einer Vielzahl von Ideen und Ansätzen beflügeln das Experimentieren und die Entwicklung neuer Konzepte.

Doch wie transportieren diese Grundrechte das Innovationsprinzip, wie setzen sie es in die Praxis um? Diesbezüglich kann man zwei Wege identifizieren: Im Rechtsstaat (vgl. Art. 2 EUV) dienen die Grundrechte als Maßstab für die Beurteilung allen staatlichen Handelns. Wenn die Staatsgewalt in den Schutzbereich eines Grundrechts eingreifen will, ist sie verpflichtet, ihr Handeln zu legitimieren, während der Träger des Grundrechts seine Handlungen oder Unterlassungen nicht zu rechtfertigen braucht, solange er innerhalb der Grenzen seines Rechts handelt. In jedem Grundrecht ist somit (theoretisch betrachtet) eine Art Regel-Ausnahmelogik verankert, die durch die Freiheit des Bürgers einerseits und die entsprechende Kompetenz der Staatsgewalten zur Einschränkung ihres Umfangs zum Schutz öffentlicher Güter andererseits definiert ist. Im Zuge der grundrechtlichen Abwehrdimension wird folglich jedes staatliche Handeln als Eingriff in die individuelle Freiheit angesehen, der in formeller Hinsicht gesetzlich und in materieller Hinsicht im Hinblick auf die Grundrechte des Bürgers 
legitimiert werden muss. ${ }^{10}$ In diesem Zusammenhang liegt die Beweislast bei den staatlichen Behörden, die regulieren wollen, sie müssen ihr „,besseres Recht“ zur Regulierung beweisen. Die Legitimationslast liegt also nicht beim Staat, weil er den Status quo ändern will, sondern weil er die durch ein bestimmtes Grundrecht geschützte individuelle Freiheit einschränken will.

Die materielle Schutzfunktion ergibt sich aus dem Zusammenspiel zwischen dem durch das jeweilige Grundrecht geschützten Gut und dem begrenzenden Gesetz, das seine konkrete Ausgestaltung in der Verhältnismäßigkeitsprüfung findet. ${ }^{11}$ Im Lichte dieses Prinzips (das sich aus der Idee der Grundrechte und ihrer Schranken sowie aus dem Rechtsstaatsprinzip ergibt) muss jedes staatliche Handeln eine dreistufige Prüfung bestehen: ${ }^{12}$ Erstens muss staatliches Handeln geeignet sein, das angestrebte Ziel zu erreichen. Zweitens muss sich das staatliche Handeln als notwendig erweisen, um das angestrebte Ziel zu erreichen. Das bedeutet, dass keine andere verfügbare Maßnahme das angestrebte Ziel auf ähnlich effektive, aber weniger freiheitseinschränkende Weise erreichen kann. Drittens: Staatliches Handeln muss angemessen sein. Dazu muss von den staatlichen Behörden ein angemessener Ausgleich zwischen dem angestrebten Ziel und dem im betreffenden Grundrecht verankerten Schutzinteresse nachgewiesen werden. Auf diese Weise unterstützen die oben genannten Grundrechte die Schaffung eines innovationsfreundlichen Ordnungsrahmens. Insofern kann man schon heute ein rechtliches „Innovationsprinzip“ erkennen, das Bestandteil der Grundrechte ist.

\section{Regulierung von Innovationen als Auftrag an den Unionsgesetzgeber}

Der bereits erwähnte Art. 3 Abs. 3 EUV nennt das Ziel der Errichtung eines Binnenmarktes. Er nennt Wirtschaftswachstum, Wettbewerbsfähigkeit und Beschäftigung als Ziele, die durch den Binnenmarkt erreicht werden sollen. Die Verwirklichung des Binnenmarktes ist jedoch untrennbar mit der Pflicht verbunden, die Umwelt, die Rechte der Verbraucher und die Gesundheit angemessen zu schützen. Art. 3 Abs. 3 EUV gibt ebenfalls das Ziel vor, einen Binnenmarkt für eine nachhaltige Entwicklung Europas und eine (wettbewerbsfähige) soziale Marktwirtschaft mit einem hohen Maß an Umweltschutz und -verbesserung zu schaffen.

Der Gesetzgeber hat alle diese Ziele zu berücksichtigen. Art. 3 Abs. 3 EUV erlaubt es nicht, einseitig auf wirtschaftlichen Nutzen und höheres Wachstum zu setzen. Gesetzliche und regulatorische Anforderungen müssen auf die Optimierung der Ergebnisse in allen Politikbereichen abzielen und ein hohes Niveau des Umwelt-, Gesundheits- und Verbraucherschutzes fordern.

Dieser Aspekt wird durch die zentralen Bestimmungen des AEU-Vertrags zum Umwelt-, Gesundheits- und Verbraucherschutz hervorgehoben:

10 BVerfGE 7, 198 (204); Sachs, in: Merten/Papier (Hrsg.), $\$ 39$, Rn. 6 ff.

11 Ausführlich dazu Ress, in: Festschrift Winkler, S. 897.

12 BVerfGE 7, 198 (205 ff.); vertiefend Alexy, S. 267 ff. 
Art. 11 AEUV besagt, dass die Erfordernisse des Umweltschutzes bei der Festlegung und Durchführung der Unionspolitiken und -maßnahmen insbesondere im Hinblick auf die Förderung einer nachhaltigen Entwicklung einbezogen werden müssen. Diese Bestimmung verlangt die Einbeziehung von Umweltschutzmaßnahmen auch in Politikbereiche, die traditionell nicht als Teil der Umweltpolitik angesehen werden. Dies wird manchmal als „Integrationsprinzip“ bezeichnet. ${ }^{13}$

Art. 191 AEUV legt ein weiteres wichtiges Prinzip des europäischen Umweltrechts fest, das für Innovationen von hoher Relevanz ist: das Vorsorgeprinzip. Art. 191 Abs. 2 AEUV wie auch Art. 114 AEUV sehen vor, dass die Umweltpolitik der EU ein hohes Schutzniveau anstrebt und auf dem Vorsorgeprinzip beruhen soll. Beim Vorsorgeprinzip geht es grundsätzlich um das Management von Risiken. Es kommt insbesondere dann zur Anwendung, wenn die wissenschaftlichen Erkenntnisse nicht eindeutig bewiesen oder zwischen Experten umstritten sind, aber eine vorläufige und objektive wissenschaftliche Risikobewertung die begründete Besorgnis aufkommen lässt, dass ein Stoff, ein Produktionsverfahren oder ein Produkt der menschlichen Gesundheit oder der Umwelt schaden könnte. ${ }^{14}$ Obwohl das Vorsorgeprinzip aus dem Umweltrecht stammt, ist es - nach der Rechtsprechung des EuGH - ein allgemeines Prinzip des EU-Rechts, das wirtschaftliche und nicht-wirtschaftliche Überlegungen einschließt. Es bietet daher geeignete Instrumente und Strategien für einen angemessenen Umgang mit Risiken.

Art. 168 AEUV besagt ebenso wie Art. 114 AEUV, dass bei der Festlegung und Durchführung aller Unionspolitiken und -maßnahmen ein hohes Gesundheitsschutzniveau sichergestellt werden muss. Nach dieser Bestimmung muss jede regulatorische Entscheidung ihre Auswirkungen auf die menschliche Gesundheit berücksichtigen, wobei ein hohes Schutzniveau zu wahren ist.

Art. 12 AEUV bekräftigt, dass den Erfordernissen des Verbraucherschutzes bei der Festlegung und Durchführung der anderen Unionspolitiken und -maßnahmen Rechnung zu tragen ist.

Art. 169 AEUV sieht vor, dass die Förderung der Interessen der Verbraucher und ein hohes Maß an Verbraucherschutz ein Ziel der Europäischen Union sind. Konkret benennt er die Gesundheit, die Sicherheit und die wirtschaftlichen Interessen der Verbraucher als Elemente des Verbraucherschutzes sowie ihr Recht auf Information, Bildung und Selbstorganisation zur Wahrung ihrer Interessen.

Die Bedeutung dieser Ziele sowie ihr rechtsverbindlicher Charakter wird durch die Charta der Grundrechte der Europäischen Union bestätigt: Der zweite Satz des Art. 35 GRCh sieht ein hohes Schutzniveau für die menschliche Gesundheit vor. Art. 37 GRCh regelt die Pflichten der Behörden zum Schutz der Umwelt. Und Art. 38 GRCh verlangt ein hohes Niveau des Verbraucherschutzes. Auch wenn umstritten ist, ob sich aus der Aufnahme dieser Bestimmungen in die Grundrechtecharta weitergehende Verpflichtungen ergeben als aus den erwähnten Bestimmungen in den EU-Verträgen, so bestätigt sie doch die gesetzliche Schutzpflicht der Union, die eben-

13 Ausführlich Calliess, in: Calliess/Ruffert, Art. 11, Rn. $1 \mathrm{ff}$.

14 Ibid., Art. 191, Rn. 26 ff. 
so wie im deutschen Recht auch den europäischen Grundrechten innewohnt, vom EuGH bislang aber noch nicht vollumfänglich entfaltet wurde. ${ }^{15}$ Sie unterstreicht auch, dass die ökologischen und sozialen Ziele gleichrangige Elemente des Binnenmarktes sind, d.h. gleichrangig mit den wirtschafts- und wachstumsbezogenen Zielen.

\section{Zwischenergebnis}

Da sich also wissenschaftlichen Akteure und Unternehmen zum Schutz ihrer Innovationsfreiheit entlang der Kette von Forschung, Entwicklung und Produktion auf die in der Europäischen Grundrechtecharta verankerten Grundrechte des Art. 13 (Wissenschaftsfreiheit), Art. 15 (Berufsfreiheit) und Art. 17 (Eigentumsrecht) berufen können, sind Innovationen seit jeher als rechtliches Schutzgut anerkannt, das in der europäischen Gesetzgebung mit den Belangen des Umweltschutzes und des Vorsorgeprinzips (vgl. Art. 191 Abs. 1 und 2 AEUV) in einen verhältnismäßigen Ausgleich zu bringen ist. Innovationen finden somit notwendig in einem regulativen Rahmen statt, innerhalb dessen auch das Vorsorgeprinzip relevant ist. Dabei ist hervorzuheben, dass Regulierung in erster Linie dazu dient, wichtige Gemeinwohlbelange zu realisieren; sie stellt somit als grundlegende Handlungsform der EU nicht nur eine Handlungsmöglichkeit, sondern einen aus den Verträgen folgenden Handlungsauftrag dar. Dies folgt für den Umweltbereich wie oben dargestellt aus Art. 11 und 191 AEUV, für den Gesundheitsschutz aus Art. 168 AEUV sowie für den Verbraucherschutz aus Art. 12 und 169 AEUV. Dabei unterliegen diese Handlungsaufträge immer den allgemeinen Anforderungen der Subsidiarität und Verhältnismäßigkeit. In diesem Rahmen ist - zuvorderst vom Gesetzgeber - im Lichte des Verhältnismäßigkeitsprinzips zu prüfen, welche Form der Regulierung eine innovationsoffene, aber zugleich vorsorgeorientierte Umweltpolitik angemessenen verwirklicht.

Wenn also manche Stimmen das Vorsorgeprinzip als Gegenprinzip zum Innovationsprinzip verstehen wollen, dann übersehen sie, dass im frühen Stadium einer neuen Technik oder eines neuen Verfahrens die Realisierung eines Risikos nicht auszuschließen ist. Insoweit stellt das Vorsorgeprinzip Verfahren und Kriterien zur Verfügung, um Risiken zu beurteilen, zu bewerten und zu managen. Ein integraler Bestandteil des Risikomanagements, wie es das Vorsorgeprinzip vorsieht, ist die Prüfung des potenziellen Nutzens und der Kosten des Handelns bzw. Nichthandelns. ${ }^{16}$ Auf dieser Basis kann vorsorgebasierte Regulierung Innovationen ermöglichen, indem gesellschaftliche Akzeptanz ausgelotet und generiert wird. Darauf wird sogleich (unter E) noch ausführlicher zurückzukommen sein.

Im Ergebnis kann Regulierung also eine Innovation zwar behindern, aber auch fördern, manchmal sogar beides gleichzeitig. Regulierung kann somit durchaus auch als Innovationsmotor wirken: So beeinflussen z.B. die allgemeine Infrastruktur, Wettbewerbs- und Beschaffungsregeln, der Zugang zu Finanzmitteln oder das Konkursrecht die Innovationsfähigkeit eines Unternehmens maßgeblich. Dies gilt auch in Be-

15 Calliess, in: Merten/Papier (Hrsg.), \44, S. 964.

16 Mitteilung der Kommission über das Vorsorgeprinzip, KOM (2001) 1 endgültig. 
zug auf den Zeitpunkt der Regulierung. Sie kann in der Phase von Forschung und Entwicklung, des Nischenmarkts oder der Marktdurchdringung erfolgen und neue, reife oder erstarrte Märkte betreffen. Zudem kann eine Nachsteuerung notwendig sein, wenn sich die regulierten Handlungsfelder verändern oder ganz neue Materien betroffen sind (z. B. die Nanotechnologie).

\section{Innovationsfreiheit, Verhältnismäßigkeit und „bessere Rechtsetzung“}

Nachdem in einem ersten Schritt der rechtliche Rahmen eines Innovationsprinzips identifiziert wurde, soll nunmehr in einem zweiten Schritt die Frage beantwortet werden, wie Innovation und Regulierung miteinander interagieren. Insoweit geht es darum, den Instrumentenpool des Rechts darauf hin zu untersuchen, welches Recht am besten geeignet ist, eine unter rechtsstaatlichen Gesichtspunkten ausgewogene Balance zwischen Innovationsoffenheit und Innovationsverantwortung zu gewährleisten. ${ }^{17}$ Vor diesem Hintergrund bleibt das klassische Ordnungsrecht unverzichtbar, es steht jedoch in dem Ruf, nicht innovationsoffen bzw. sogar innovationshemmend zu sein, indem es z.B. zur Risikovorsorge oder Gefahrenabwehr der gesellschaftlichen Innovation Grenzen setzt. Gleichwohl erkennt die rechtswissenschaftliche Innovationsforschung an, dass imperatives Recht auch Innovationen stimulieren kann. ${ }^{18}$

Schon damit wird deutlich, dass innovationsfördernde Regulierung nur auf den ersten Blick einen In-Sich-Widerspruch formuliert. Zwar verweist Innovation auf das Unbekannte, das eben noch nicht Existente und überraschend Neue, während Regulierung, gleichgültig wie man sie im Einzelnen definieren möchte, durch die Verfolgung eines bestimmten Zweckes gekennzeichnet ist, also der Realisierung eines bekannten oder jedenfalls konkret gedachten Zielzustandes dient. Jedoch wird Innovationsförderung von der Rechtsordnung nicht nur in einer Basisschicht durch die Sicherung von Freiheit gewährleistet, sondern vielmehr kann Freiheit allein die Innovationsfähigkeit einer Gesellschaft mit Blick auf die sozialen Verbundenheiten und die wechselseitigen Abhängigkeiten von Individuen und Organisationen nur in bestimmten (rechtlichen) Grenzen sichern. Insoweit geht es um eine rechtliche Infrastruktur, die verfassungsrechtlichen, konkret rechtsstaatlichen, Maßstäben genügt. Was aber sind genau die rechtsstaatlichen Vorgaben für die gesuchte rechtliche Infrastruktur?

In diesem Zusammenhang ist die europäische Agenda für bessere Rechtsetzung (Better Regulation Agenda) ${ }^{19}$ von Interesse, die zu einer Priorität in der Arbeit der

17 Hoffmann-Riem, Innovationsoffenheit und Innovationsverantwortung, AöR 2006/2, S. 271.

18 Ibid., S. 272.

19 Europäische Kommission, Mitteilung der Kommission an das Europäische Parlament, den Europäischen Rat und den Rat „Bessere Rechtsetzung: Bessere Ergebnisse für eine stärkere Union“, $\mathrm{KOM}(2016) 615$ final vom 14.9.2016, S. 2; ferner: Bestandsaufnahme der Agenda für bessere Rechtsetzung vom 15.4.2019, COM(2019) 178; sowie Jahresbericht 2018 über die Anwendung der Grundsätze der Subsidiarität und der Verhältnismäßigkeit 2018, $\operatorname{COM}(2019) 333$, S. 4 ff. und Jahresbericht 2019, $\operatorname{COM}(2020) 272$, S. 3 ff. 
Europäischen Kommission geworden ist. Der rechtliche Rahmen für die Politik der besseren Rechtsetzung ergibt sich aus den Grundsätzen der Subsidiarität und der Verhältnismäßigkeit, die in Art. 5 Abs. 3 und Abs. 4 EUV festgelegt sind. Das Subsidiaritätsprinzip sieht vor, dass die EU ihre nicht ausschließlichen Befugnisse ausüben kann, wenn (1) die Mitgliedstaaten die geplante Maßnahme nicht in ausreichendem Maße durchführen können und (2) die Maßnahme von der Union vergleichsweise besser durchgeführt werden kann. Insofern zielt das Subsidiaritätsprinzip darauf ab, unwirksame, unnötige und damit belastende Regelungen auf Unionsebene zu begrenzen. Der Grundsatz der Verhältnismäßigkeit (Art. 5 Abs. 4 EUV) sieht vor, dass die Maßnahmen der Union inhaltlich und formal nicht über das hinausgehen dürfen, was zur Erreichung der Ziele der Verträge erforderlich ist. Obwohl der historische Hintergrund des Grundsatzes der Verhältnismäßigkeit der Schutz der Grundrechte bzw. der vier Grundfreiheiten vor öffentlichen Handlungen ist, ist er auch für die Agenda für bessere Rechtsetzung und die Förderung von Innovation durch intelligente Regulierung relevant.

Hier, im rechtsstaatlichen Grundsatz der Verhältnismäßigkeit, ist die Agenda für bessere Rechtsetzung zu verorten. Ein gesetzgeberischer Werkzeugkasten („Legislative Toolbox") würde einer flexibleren und insoweit die Kompetenzen der Mitgliedstaaten stärker schonenden europäischen Gesetzgebung den Weg bereiten: ${ }^{20}$ Die Werkzeuge könnten von der gegenseitigen Anerkennung auf der Grundlage des Herkunftslandprinzips bis hin zu strikter Harmonisierung durch die Setzung von Standards reichen. Zwischen diesen beiden Extremen könnten verschiedene Formen der Rechtsetzung genutzt werden. So könnten europäische Rechtsakte entweder der nationalen Ebene eine Berücksichtigung alternativer, weniger belastender Lösungen erlauben oder sich stärker auf Ergebnisse konzentrieren, anstatt detailliert die Maßnahmen vorgeben. Auch könnten Rechtsakte ein sog. „Anfechtungsrecht“ enthalten, das es nationalen Behörden ermöglicht, bei der Kommission eine Ausnahme von einer existierenden Regel oder Bestimmung zu beantragen. Schließlich könnten europäische Rechtsakte, die komplexe und noch ungewisse Sachverhalte betreffen, mit sog. „Sunset Clauses" versehen werden, die insoweit eine Ähnlichkeit zu experimentellen Gesetzen aufweisen, als sie es dem Gesetzgeber ermöglichen, einen neuen regulatorischen Ansatz „auszuprobieren“. In diesem Zusammenhang könnten auch Werkzeuge wie „Benchmarking“ und „Best Practice“ zur Anwendung kommen, im Zuge derer eine vergleichende Bewertung und darauf basierend die Identifizierung des besten mitgliedstaatlichen Regulierungsansatzes ermöglicht wird, der dann zum europäischen Maßstab werden kann.

Bessere Rechtsetzung ist freilich kein Selbstzweck, sie muss dem Gemeinwohl dienen. Dieses ist im europäischen Staaten- und Verfassungsverbund, ${ }^{21}$ der im Hinblick auf den primär in mitgliedstaatlicher Kompetenz liegenden Vollzug des Unionsrechts

20 Zum Kontext Calliess, integration 2019/2, S. $111 \mathrm{ff}$.

21 Dazu Calliess, Die neue Europäische Union, S. $47 \mathrm{ff}$. 
zugleich als Verwaltungsverbund beschrieben wird,22 durch die vorstehend geschilderten verfassungsrechtliche Rahmenvorgaben mitbestimmt.

\section{E. Gemeinwohlverträgliches Innovationspotential durch Recht ermöglichen}

Anknüpfungspunkt für staatliche Regulierung im Interesse von Belangen des Gemeinwohls ist im europäischen Verwaltungsverbund die behörliche Genehmigung. Sie ist das verwaltungsrechtliche Instrument, mit dem die verfassungsrechtlichen Vorgaben samt Verhältnismäßigkeitsprüfung in das einfache Recht transportiert und transformiert werden können. Sie ist somit ein Hebel, mit dem im europäischen Staaten- und Verfassungsverbund Innovationsanreize im Interesse bestimmter, verfassungsrechtlich legitimierter öffentlicher Belange (z.B. Umwelt-, Verbraucher-, Gesundheitsschutz) gesetzt werden können. Insoweit wird, abstrakt durch den Gesetzgeber und konkret durch die Behörden „Freiheit verteilt“.23

Aus der rein bipolaren Perspektive des dogmatisch durch die grundrechtliche Abwehrdimension bestimmten Verfassungsrechtsverhältnisses zwischen Wirtschaft und Staat stellt sich das Recht, konkret die verwaltungsrechtliche Genehmigung, zwar oftmals als Investitions- und Innovationshindernis dar. Jedoch wird dabei eine wichtige Funktion des Rechts, die sich aus der Perspektive mehrpoliger Verfassungsrechtsverhältnisse ergibt, übersehen. Diese besteht darin, die Entfaltung unternehmerischer Freiheit (Art. 15, 17 GRCh) in einem demokratischen Rechtsstaat überhaupt erst zu ermöglichen. Aus der Sicht der Grundrechte des Unternehmers erfüllt Recht damit auch die Funktion, die rechtlichen und organisatorischen Voraussetzungen seiner Freiheitsausübung zu sichern. Im Umweltrecht geschieht dies z.B. dadurch, dass die staatliche Genehmigung die Funktion einer Standardisierung und Marktermöglichung hat und zugleich die rechtlichen Entfaltungsvoraussetzungen der Wirtschaftsfreiheit einschließlich der Gewährleistung von Rechtssicherheit gewährleistet. ${ }^{24}$

Diese Ermöglichungsfunktion des Rechts kommt insbesondere dort, wo die grundrechtlichen Schutzpflichten des Staates gegenüber den Betroffenen aktuell werden, zum Ausdruck. Insoweit leistet öffentlich-rechtliche Regulierung einen entscheidenden Beitrag zur Entfaltung der Wirtschaftsfreiheit, wenn sie durch Ausschluss privatrechtlicher Abwehransprüche bei staatlich genehmigten Aktivitäten ${ }^{25} \mathrm{im}$ Wege von Präklusionsregelungen dem Begünstigten Rechtssicherheit gegenüber den Betroffenen verschafft. Über die staatliche Zulassungsentscheidung gewinnt der Begünstigte darüber hinaus die für die Entfaltung der Wirtschaftsfreiheit unerlässliche Investitionssicherheit. ${ }^{26} \mathrm{Um}$ eine Genehmigung zu erlangen, ist der durch sie Begünstigte vor diesem Hintergrund also in aller Regel bereit, Kompromisse einzugehen und zu kooperieren.

22 Zum Ganzen Schmidt-Aßmann, DVBl 1993, S. 925 f.; Schmidt-Aßmann/Schöndorf-Haubold (Hrsg.); Weiß; Kabl, Der Staat 2011/3, S. 353 ff. m.w.N.

23 Zur Ambivalenz dessen Calliess, Rechtsstaat und Umweltstaat, S. $373 \mathrm{ff}$.

24 Vgl. Kloepfer, NuR 1997, S. 417 f.

25 Grundlegend Sach.

26 Dazu Calliess, Rechtsstaat und Umweltstaat, S. 264 ff. und insbesondere S. $384 \mathrm{ff}$. 
Hier kann nunmehr an den geschilderten Zusammenhang zwischen Innovationsprinzip, innovationsoffener Regulierung und Verhältnismäßigkeitsprüfung angeknüpft werden.

\section{Das Zusammenspiel von Genehmigung, Verhältnismäßigkeits- und Alternativenprüfung}

Interessant ist in diesem Zusammenhang vor allem die Verbindung von Verhältnismäßigkeits- und Alternativenprüfung. Wie vorstehend gezeigt wurde ist die Alternativenprüfung ein zentrales Instrument der besseren Rechtssetzung, mit Hilfe dessen innovationsoffene Regulierung ermöglicht werden kann.

Die Alternativenprüfung weist Parallelen zur Verhältnismäßigkeitsprüfung, insbesondere zu deren zweiter Stufe, der Erforderlichkeitsprüfung auf. ${ }^{27}$ Zum einen geht es in beiden Fällen darum, unter mehreren, zur Erreichung eines Zieles geeigneten Mitteln das am wenigsten eingreifende zu suchen. Gegenüber der Verweigerung der Genehmigung oder dem Erlass noch strengerer Auflagen kann sich die Prüfung und Erörterung von Alternativen mit Blick auf die Einhaltung der nach dem Vorsorgeprinzip gebotenen Umweltqualitätsnormen ${ }^{28}$ für den Antragsteller durchaus als milderes Mittel darstellen, dass nicht zuletzt in seinem Interesse liegt. Zum anderen ist das Denken in Alternativen ein wesentlicher Bestandteil der Verhältnismäßigkeitsprüfung insgesamt, und zwar sowohl bei der Suche nach den geeigneten Mitteln (Zwecktauglichkeit), als auch bei der Suche nach einem gleich wirksamen milderen Mittel (Erforderlichkeit), wie auch bei der Suche nach der angemessenen Zweck-Mittel-Relation (Proportionalität). Dies liegt in der Tatsache begründet, dass das allgemeine Schema der Alternativenprüfung ebenso wie das Verhältnismäßigkeitsprinzip eine Ausformung der Struktur zweckrationalen Handelns darstellt: Ein Akteur setzt sich ein Ziel, ersinnt verschiedene Mittel der Zielerreichung, und wählt dasjenige aus, das einerseits das Ziel am besten erreicht, mithin am geeignetsten ist, andererseits die geringsten nachteiligen Nebenfolgen hat. Sind die Nachteile dieses Mittels im Verhältnis zu den Vorteilen, die das Ziel verspricht, unangemessen hoch, empfiehlt sich ein Verzicht auf das Ziel.

\section{Die Alternativenprüfung als Instrument einer innovationsoffenen Regulierung im europäischen Umweltrecht}

Eine Pflicht zur Prüfung von alternativen Produkten (Substituten) besteht z.B. in der Verordnung zur Registrierung, Bewertung, Zulassung und Beschränkung chemischer Stoffe (REACH-VO). ${ }^{29}$

27 Winter, S. 27 ff. und S. 43 ff.; vgl. auch Volkmann, VerwArch 1998, S. 392; HoffmannRiem, DVBl. 1994, S. $608 \mathrm{f}$.

28 Calliess, Rechtsstaat und Umweltstaat, S. $235 \mathrm{ff}$.

29 VO (EG) Nr. 1907/2006 zur Registrierung, Bewertung, Zulassung und Beschränkung chemischer Stoffe (REACH), ABl. L 396 v. 30.12.2006, S. 1. 
Als Zweck der Zulassungsvorschriften (Teil VII) bestimmt die REACH-VO in ihrem Art. 55 REACH neben der Funktionsfähigkeit des Binnenmarktes und der Beherrschung der stofflichen Risiken,

„dass die [besonders besorgniserregenden] Stoffe schrittweise durch geeignete Alternativstoffe oder - technologien ersetzt werden, sofern diese wirtschaftlich und technisch tragfähig sind. Zu diesem Zweck prüfen alle Hersteller, Importeure und nachgeschalteten Anwender, die einen Antrag auf Zulassung stellen, die Verfügbarkeit von Alternativen und deren Risiken sowie die technische und wirtschaftliche Durchführbarkeit der Substitution."

Zulassungsvoraussetzung ist nach Art. 60 Abs. 2 REACH, dass das mit der Verwendung des Stoffes verbundene Risiko für die menschliche Gesundheit oder die Umwelt, das sich aus seinen inhärenten Eigenschaften ergibt, angemessen beherrscht wird. Sollten diese Anforderungen nicht erfüllt oder nicht erfüllbar sein, ist eine Zulassung nach Art. 60 Abs. 4 S. 1 REACH nur möglich,

„wenn nachgewiesen wird, dass der sozioökonomische Nutzen die Risiken überwiegt, die sich aus der Verwendung des Stoffes für die menschliche Gesundheit oder die Umwelt ergeben, und wenn es keine geeigneten Alternativstoffe oder-technologien gibt."

Der Zulassungsantrag hat gem. Art. 62 Abs. 4 REACH zu enthalten:

„e) eine Analyse der Alternativen unter Berücksichtigung ihrer Risiken und der technischen und wirtschaftlichen Durchfübrbarkeit der Substitution, gegebenenfalls einschließlich Informationen über einschlägige Forschungs- und Entwicklungstätigkeiten des Antragstellers;

f) sofern die unter Buchstabe e genannte Analyse erweist, dass [...] geeignete Analysen verfügbar sind, einen Substitutionsplan einschließlich eines Zeitplans für die vom Antragsteller vorgeschlagenen Maßnahmen.“

Aus den genannten Vorschriften ergibt sich demnach eine Verpflichtung des Antragstellers zur Suche nach geeigneten Alternativen, die allerdings auf den Fall beschränkt ist, dass dieser im Einzelfall den Nachweis der angemessenen Beherrschung des Risikos nicht führen kann. ${ }^{30}$

Hinsichtlich der Alternativenprüfungspflicht der Zulassungsbehörde bestimmt Art. 60 Abs. 4 S. 2 REACH, dass die Entscheidung unter Berücksichtigung folgender Aspekte zu treffen ist:

„c) Analyse der vom Antragsteller (...) vorgelegten Alternativen oder eines vom Antragsteller $(\ldots)$ vorgelegten Substitutionsplans;

d) verfügbare Informationen über die Risiken für die menschliche Gesundheit oder die Umwelt von Alternativstoffen oder -technologien."

Weiter bestimmt Art. 60 Abs. 5 REACH:

„Bei der Beurteilung, ob geeignete alternative Stoffe oder Technologien verfügbar sind, berücksichtigt die Kommission alle maßgeblichen Aspekte einschließlich der folgenden:

30 Calliess/Lais, NuR 2005, S. 297. 


\begin{abstract}
a) die Frage, ob der Übergang zu Alternativen zu einem geringeren Gesamtrisiko für die menschliche Gesundheit und die Umwelt führen würde, wobei der Angemessenheit und Wirksamkeit von Risikomanagementmaßnahmen Rechnung zu tragen ist;

b) die technische und wirtschaftliche Durchfübrbarkeit der Alternativen für den Antragsteller."
\end{abstract}

Die Prüfungspflicht der Zulassungsbehörde ist demnach keineswegs auf eine Betrachtung der vom Antragsteller vorgelegten Alternativen begrenzt. Sie wird bezüglich der Beurteilung möglicher Alternativen dennoch regelmäßig auf das Know-how bzw. den Wissensvorsprung der jeweiligen Antragsteller angewiesen sein.

Im Ergebnis ist eine Alternativenprüfung also grundsätzlich geeignet, Innovationen zu fördern. ${ }^{31}$ Freilich sind dafür bestimmte Voraussetzungen notwendig, wie das Beispiel des Chemikalienrechts unten den Aspekten der verpflichtenden Ausgestaltung bis hin zum Substitutionsziel deutlich macht.

\title{
III. Weitergehende Perspektiven einer Kopplung von Innovation und Alternativenprüfung
}

Es lohnt sich jedoch im Interesse von Innovationsoffenheit und Innovationsverantwortung vorstehend genannte Defizite zu überwinden. Denn das Instrument der Alternativenprüfung bietet nicht nur für den Vorhabensträger, der im Rahmen der Untersuchung alternativer Projektverwirklichungsmöglichkeiten den für ihn vorteilhaftesten Weg ermitteln kann, Chancen, sondern kann auch auf Seiten des Staates eingesetzt werden, um technische Entwicklung zu forcieren und in eine bestimmte Richtung zu lenken. ${ }^{32}$ Innovationen, d.h. neue Produkte, Techniken und Verfahren werden als zentraler Gegenstand und maßgebliche Herausforderung des Umweltrechts verstanden, denn die schwierige Aufgabe des Umweltrechts besteht darin, steuernd und regulierend die vielfältigen Risiken technischer, ökonomischer und gesellschaftlicher Entwicklungen auf umwelt- und gemeinwohlverträglichem Niveau zu halten, ohne die wirtschaftliche Entwicklung unnötig zu beeinträchtigen. ${ }^{33}$

Die folgenden Ausführungen beschränken sich auf das legislative Instrument der Innovationssteuerung (Gesetzgebung), d.h. die Möglichkeiten der Steuerung durch die Verwaltung (insb. Subventionen) sowie die Rechtsprechung werden hier ausgeklammert. ${ }^{34} \mathrm{Zu}$ prüfen ist, wie die gesetzlichen Regelungen ausgestaltet sein müssen, damit für die Normadressaten, d.h. die Vorhabensträger, ein Anreiz zur Entwicklung und Anwendung fortschrittlicher, umweltschonender Alternativtechniken und -stoffe geschaffen werden kann.

31 Hoffmann-Riem, DV 2005, S. 156.

32 Winter, S. 26.

33 Koch, in: Hoffmann-Riem/Schneider (Hrsg.), S. 273.

$34 \mathrm{Zu}$ den Instrumenten der Innovationssteuerung vgl. Schulze-Fielitz, in: Hoffmann-Riem/ Schneider (Hrsg.), S. $296 \mathrm{ff}$. 


\section{Förderung von technischer und stofflicher Innovation}

Insoweit lassen sich mit Blick auf die Regulierungsmöglichkeiten des Gesetzgebers zwei Arten der Innovationssteuerung unterscheiden. Er kann zum einen - positiv für einen künftigen Zeitpunkt einen Zielwert (z.B. einen Qualitätsstandard, Emissionsgrenzwert etc.) festlegen, der nach Ablauf der Übergangsfrist einzuhalten ist und somit technische Innovation provoziert. Zum anderen kann - negativ - unerwünschten Techniklinien bzw. Stoffen eine Auslaufzeit gewährt werden, nach deren Ablauf eine Alternative entwickelt sein muss (vgl. sog. phasing-out von FCKW innerhalb gesetzter Frist). ${ }^{35}$

Konkret wäre es zum Beispiel mit Blick auf eine Innovationsförderung im Bereich der technischen und stofflichen Entwicklung in Anlehnung an das (im japanischen Energieeffizienzrecht entwickelte) sog. „Top-Runner-Modell“ denkbar, dass sich das beantragte Vorhaben einem Vergleich mit dem zum Antragszeitpunkt fortschrittlichsten stofflichen bzw. technischen Standard stellen muss. Im Anschluss an eine Alternativenprüfung könnte eine rückständige Techniklinie nur noch mit der Maßgabe genehmigt werden, dass innerhalb einer Übergangsfrist der Top-Runner-Standard erreicht wird. Die Praktikabilität dieses Lösungsvorschlags ist - zugegebenermaßen - aufgrund des häufigen Aktualisierungsbedarfs des untergesetzlichen Regelwerks und der Vielfältigkeit der Techniklinien und Stoffe nicht einfach zu gewährleisten.

\section{Alternativenprüfung de lege ferenda}

Die Alternativenprüfung kann über die Genehmigung - bzw. die Möglichkeit der Genehmigungsversagung - beim Vorhabensträger Innovationsanreize setzen. Sie kann vom Gesetzgeber in unterschiedlicher Form ausgestaltet werden. ${ }^{36}$

- Sie kann nur prozedural festgelegt werden, mit der Folge, dass nur der Vorgang der Prüfung an sich durch die Behörde kontrolliert werden kann, die Wahl einer Alternative im Ergebnis aber völlig frei bleibt und von der Behörde nicht beeinflusst werden kann.

- Sie kann aber auch durch die Vorgabe bestimmter Topoi, die beachtet werden müssen, inhaltlich gesteuert werden. Insoweit ist die Behörde auf eine nachvollziehende Kontrolle beschränkt, bei der ein Auswahlspielraum des Vorhabensträgers anerkannt wird.

- Des Weiteren ist eine Gestaltung der Alternativenprüfung denkbar, nach der die Behörde eine Ergebniskontrolle vorzunehmen hat, die sie befugt, die Prüfung des Vorhabensträgers im Ergebnis nachzuprüfen und dabei Defizite festzustellen. Ist

35 Winter, S. 26, $46 \mathrm{f}$.

36 Vgl. Calliess, Rechtsstaat und Umweltstaat, S. 595 f.: Soweit statt einer abschlägigen Entscheidung eine umweltverträglichere zulassungsfähige Alternative in Frage kommt, ist diese im Sinne des Verhältnismäßigkeitsgrundsatzes, genauer der Erforderlichkeit, als milderes Mittel zu betrachten. 
dies der Fall, darf die Behörde den Antrag mit der Begründung ablehnen, dass eine vom Vorhabensträger nicht gewählte Variante vorzugswürdig ist. Die Ergebniskontrolle könnte aber auch darin bestehen, dass die Behörde eine vom Vorhabensträger nicht selbst eingebrachte vorzugswürdige Alternative genehmigt.

In der Regel wird all dies nicht durch behördliche Auflagen zu bewerkstelligen sein, so dass die Bereitschaft des Vorhabensträgers, seinen Antrag entsprechend zu ändern, zur Voraussetzung wird. Andernfalls würde er in einer mit der grundrechtlich garantierten Wirtschaftsfreiheit nicht zu vereinbaren Weise zu einer Investition gezwungen. Auch wenn die Einräumung von Versagungsermessen verfassungsrechtlich möglich ist ${ }^{37}$ so stellt sie doch einen weitreichenden Eingriff in die Wirtschaftsfreiheit des Begünstigten (mithin des Vorhabensträger) dar, die mit Blick auf das Übermaßverbot nur schwer gerechtfertigt werden kann. Ein im Hinblick hierauf milderes Mittel stellt die verpflichtende Alternativenprüfung dar. Vor diesem Hintergrund erscheint eine Objektivierung der Alternativenprüfungspflicht auf Seiten des Antragstellers möglich und erforderlich. Einer Pflicht zur Durchführung einer Alternativenprüfung stünden daher keine durchgreifenden verfassungsrechtlichen Bedenken entgegen.

\section{F. Ausblick}

Wenn sich wissenschaftlichen Akteure und Unternehmen zum Schutz ihrer Innovationsfreiheit entlang der Kette von Forschung, Entwicklung und Produktion auf die in der Europäischen Grundrechtecharta verankerten Grundrechte des Art. 13 (Wissenschaftsfreiheit), Art. 15 (Berufsfreiheit) und Art. 17 (Eigentumsrecht) berufen können, sind Innovationen seit jeher als rechtliches Schutzgut anerkannt, das in der europäischen Gesetzgebung mit den Belangen des Umweltschutzes und des Vorsorgeprinzips (vgl. Art. 191 Abs. 1 und 2 AEUV) in einen verhältnismäßigen Ausgleich zu bringen ist. Hieran würde die Einführung eines eigenständigen Innovationsprinzips nichts ändern, da ein Prinzip im Recht eine geringere Wirkkraft als individuelle Grundrechte entfaltet.

Innovationen finden somit notwendig in einem regulativen Rahmen statt, innerhalb dessen auch das Vorsorgeprinzip relevant ist. Dabei ist hervorzuheben, dass Regulierung in erster Linie dazu dient, wichtige Gemeinwohlbelange zu realisieren; sie stellt somit als grundlegende Handlungsform der EU nicht nur eine Handlungsmöglichkeit, sondern einen aus den Verträgen folgenden Handlungsauftrag dar. Dies folgt für den Umweltbereich wie oben dargestellt aus Art. 11 und 191 AEUV, für den Gesundheitsschutz aus Art. 168 AEUV sowie für den Verbraucherschutz aus Art. 12 und 169 AEUV.

Gerade die enormen Herausforderungen der Gegenwart - Digitalisierung und Dekarbonisierung - verlangen tiefgreifende Innovationen. Wenn das Innovationsprinzip aus rechtlicher Perspektive wie gezeigt nicht als Gegenprinzip zum Vorsorgeprinzip verstanden werden darf, kann es allein darum gehen, die Offenheit für Innovation in allen Phasen des Politikzyklus zu erhöhen. Auf dieser Basis kann das Innovationsprin- 
zip dazu beitragen, die europäische Gesetzgebung innovationsoffen auszugestalten. Regulierung wird aus dieser Perspektive allerdings zu Unrecht allein als Hemmnis für Innovationen betrachtet. Schon aus wirtschaftlicher Sicht genügt es nicht, Innovationsprozesse zu fördern, sondern vielmehr ist gerade in der Übergangsphase der Innovation von der Nische in den breiten Markt eine flexible politisch-regulative Steuerung durch den Staat erforderlich, die einen stabilen Rahmen schafft, der den Unternehmen die notwendige Planungs- und Investitionssicherheit vermittelt. Dies geschieht überwiegend durch Regulierung, die daher auch ein Treiber von Innovationen sein kann: Nicht zuletzt können durch Regulierung erwünschte Innovationen angereizt und gelenkt werden, um wichtige Gemeinwohlziele wie etwa den Umweltschutz zu fördern. Eine solche Perspektive ist nicht von ungefähr auch Grundlage des European Green Deal. ${ }^{38}$

In diesem Rahmen ist - zuvorderst vom Gesetzgeber - im Lichte des Verhältnismäßigkeitsprinzips zu prüfen, welche Form der Regulierung eine innovationsoffene, aber zugleich vorsorgeorientierte Umweltpolitik angemessenen verwirklicht. Die Ermöglichung von Alternativenprüfungen im Rahmen der Verhältnismäßigkeitsprüfung kann Räume für Innovationen schaffen. Daran anknüpfend kann experimentelle Regulierung, die beispielsweise für bestimmte Tätigkeiten und zeitlich begrenzt eine Abweichung von bestehenden Standards zulässt, innovative Ansätze befördern. Regulierung kann im Ergebnis also auch als Innovationsmotor wirken, was insbesondere aus dem Wechselspiel zwischen dem Wettbewerb mit Regeln und Praktiken des bestehenden Feldes einerseits und der Beeinflussung von politischen Zielvorgaben und Maßnahmen, technologischer Innovation und Marktdynamik andererseits geschieht. Ein Beispiel sind insoweit sogenannte Top-Runner-Regulierungen, die in regelmäßigen Abständen das energie- oder ressourceneffizienteste Produkt zum neuen, für die ganze Branche zu erreichendem Standard erklären.

\section{BIBLIOGRAPHIE}

ALEXY, ROBERT, Theorie der Grundrechte, Berlin, 1986

CALLIESS, CHRISTIAN, Rechtsstaat und Umweltstaat, München, 2001

CALLIESS, CHRISTIAN, in: Merten, Detlef; Papier, Hans-Jürgen (Hrsg.), Handbuch der Grundrechte in Deutschland und Europa, Heidelberg, 2006

CALLIESS, CHRISTIAN, Die neue Europäische Union nach dem Vertrag von Lissabon, Tübingen, 2010

CALLIESS, CHRISTIAN; RUFFERT, MATTHIAS, EUV/AEUV-Kommentar, 5. Aufl., München, 2016

CALLIESS, CHRISTIAN, Zukunftsszenarien und Reformoptionen für die Europäische Union: vom Weißbuch der Kommission zu einer flexibleren Arbeitsmethode, integration 2/2019, S. 97-117

38 Dazu ausführlich Calliess/Dross, ZUR 2020, S. $456 \mathrm{ff}$. 
CALLIESS, CHRISTIAN; DROSS, MIRIAM, Umwelt- und Klimaschutz als integraler Bestandteil der Wirtschaftspolitik - Überlegungen im Lichte von European Green Deal und Corona-Recovery Plan, Zeitschrift für Umweltrecht (ZUR), 2020, Heft 9, S. $456 \mathrm{ff}$.

CALLIESS, CHRISTIAN; LAIS, MARTINA, REACH revisited - Der Verordnungsvorschlag zur Reform des Chemikalienrechts als Beispiel einer neuen europäischen Vorsorgestrategie, Natur und Recht (NuR), 2005, Jg. 27(5), S. 290-299

GRANIERI, MASSIMILIANO; RENDA, ANDREA, Innovation Law and Policy in the European Union: Towards Horizon 2020, Heidelberg, 2012

HOFFMANN-RIEM, WOLFGANG, Von der Antragsbindung zum konsensierten Optionenermessen, Deutsches Verwaltungsblatt (DVBl.), 1994, S. 605 ff.

HOFFMANN-RIEM, WOLFGANG, Risiko- und Innovationsrecht im Verbund, Deutsche Verwaltung (DV), 2005, Jg. 38, S. $145 \mathrm{ff}$.

HOFFMANN-RIEM, WOLFGANG, Innovationsoffenbeit und Innovationsverantwortung, Archiv des öffentlichen Rechts (AöR), 2006, Jg. 131(2), S. 255-277

KAHL, WOLFGANG, Der Europäische Verwaltungsbund: Strukturen - Typen Phänomene, 2011, Jg. 50(3), S. 353-388

KLOEPFER, MICHAEL, Recht ermöglicht Technik, Natur und Recht (NuR), 1997, S. $417 \mathrm{f}$.

KOCH, HANS-JOACHIM, Innovationssteuerung im Umweltrecht, in: HoffmannRiem, Wolfgang; Schneider, Jens-Peter (Hrsg.), Rechtswissenschaftliche Innovationsforschung, Baden-Baden, 1998, S. 273-290

MARCHANT GARY E; ALLENBY, BRADEN R; HERKERT, JOSEPH R (Hrsg.), The Growing Gap Between Emerging Technologies and Legal-Ethical Oversight: The Pacing Problem, Heidelberg, 2011

RESS, GEORG, Menschenrechte, europäisches Gemeinschaftsrecht und nationales Verfassungsrecht, in: Staat und Recht. Festschrift für Günther Winkler, Wien, 1997

SACH, KARSTEN, Genebmigung als Schutzschild?, Köln, 1994

SACHS, MICHAEL, Abwehrrechte, in: Merten, Detlef; Papier, Hans-Jürgen (Hrsg.), Handbuch der Grundrechte in Deutschland und Europa, Band II, München, 2006, $\$ 39$

SCHMIDT-AßMANN, EBERHARD, Deutsches und Europäisches Verwaltungsrecht - Wechselseitige Einwirkungen, Deutsches Verwaltungsblatt (DVB1.), 1993, S. $924 \mathrm{ff}$.

SCHMIDT-AßMANN, EBERHARD; SCHÖNDORF-HAUBOLD, BETTINA (Hrsg.), Der Europäische Verwaltungsverbund, Tübingen, 2005

SCHULZE-FIELITZ, HELMUTH, Instrumente der Innovationssteuerung durch Öffentliches Recht, in: Hoffmann-Riem, Wolfgang; Schneider, Jens-Peter (Hrsg.), Rechtswissenschaftliche Innovationsforschung. Grundlagen, Forschungsansätze, Forschungsfelder, Baden-Baden, 1998, S. 291-329 
VOLKMANN, UWE, Umweltrechtliches Integrationsprinzip und Vorhabengenehmigung, Verwaltungsarchiv (VerwArch), 1998, Jg. 89(3), S. 363-399

WEIß, WOLFGANG, Der Europäische Verwaltungsverbund - Grundfragen, Kennzeichen, Herausforderungen, Berlin, 2010

WINTER, GERD, Alternativen in der administrativen Entscheidungsbildung, Düsseldorf, 1997 